\title{
Vicarious experiences and detection accuracy while observing pain and touch: The effect of perspective taking
}

\author{
S. Vandenbroucke ${ }^{1} \cdot$ G. Crombez $^{1} \cdot$ T. Loeys ${ }^{2} \cdot$ L. Goubert $^{1}$
}

Published online: 2 April 2015

(C) The Psychonomic Society, Inc. 2015

\begin{abstract}
In this study, we investigated the effects of observing pain and touch in others on vicarious somatosensory experiences and the detection of subtle somatosensory stimuli. Furthermore, the effect of taking a first- versus a third-person perspective was investigated. Undergraduates $(N=57)$ viewed videos depicting hands being pricked (pain), hands being touched by a cotton swab (touch), and control scenes (same approaching movement of a hand as in the other video categories, but without the painful/touching object) while experiencing vibrotactile stimuli themselves on the left, on the right, or on both hands. Participants reported the location at which they felt a somatosensory stimulus. The vibrotactile stimuli and visual scenes were applied in a spatially congruent or incongruent way, and other trials were presented without vibrotactile stimuli. The videos were depicted in first-person perspective and third-person perspective (i.e., the videos were shown upside down). We calculated the proportions of correct responses and false alarms (i.e., numbers of trials on which a vicarious somatosensory experience was reported congruent or incongruent to the site of the visual information). Painrelated scenes facilitated the detection of tactile stimuli and augmented the number of vicarious somatosensory experiences, as compared with observing the touch or control videos. Detection accuracy was higher for videos depicted in first-person perspective than for those in third-person
\end{abstract}

S. Vandenbroucke

Sophie.Vandenbroucke@ugent.be

1 Department of Experimental-Clinical and Health Psychology, Ghent University, Henri Dunantlaan, 2, Ghent 9000, Belgium

2 Department of Data Analysis, Ghent University, Ghent, Belgium perspective. Perspective had no effect on the number of vicarious somatosensory experiences. This study indicates that somatosensory detection is particularly enhanced during the observation of pain-related scenes, as compared to the observation of touch or control videos. These research findings further demonstrate that perspective taking impacts somatosensory detection, but not the report of vicarious experiences.

Keywords Touch $\cdot$ Multisensory processing $\cdot$ Modularity of perception $\cdot$ Vicarious pain $\cdot$ Vicarious touch

Our senses do not operate independently (Spence \& Driver, 2004). For example, tactile perception is facilitated when viewing the body. Such findings suggest a strong link between vision and somatosensation (Kennett, Taylor-Clarke, \& Haggard, 2001). Also, observing somatosensory stimuli being applied to another person influences the detection of tactile stimuli in the observer (Cardini, Haggard, \& Làdavas, 2013; Gillmeister, 2014; Vandenbroucke, Crombez, Harrar, et al., 2014; Vandenbroucke, Crombez, Loeys, \& Goubert, 2014). In line with this finding, brain-processing studies have shown that somatosensory activity is enhanced when observing bodily sensations in others (Blakemore, Bristow, Bird, Frith, \& Ward, 2005; Ebisch et al., 2008; Keysers, Kaas, \& Gazola, 2010; Schaefer, Heinze, \& Rotte, $2005,2012)$. An extreme variant of the modulation of somatosensory detection by observing touch or pain is the actual experience of such sensations when no stimulus is presented ("vicarious somatosensory experiences"). Vicarious somatosensory experiences are intriguing, since they indicate that tactile or nociceptive input may not be necessary to experience touch or pain (Fitzgibbon, Giummarra, Georgiou-Karistianis, Enticott, \& Bradshaw, 2010). 
Little systematic research is available on the occurrence of vicarious somatosensory experiences and the factors affecting this phenomenon (Fitzgibbon et al., 2012; Fitzgibbon, Giummarra, et al., 2010; Osborn \& Derbyshire, 2010; Vandenbroucke, Crombez, Harrar, et al., 2014; Vandenbroucke et al., 2013). Vandenbroucke et al. (2013; Vandenbroucke, Crombez, Loeys, \& Goubert, 2014) showed that individuals reporting vicarious pain in daily life ("pain responders") reported more vicarious somatosensory experiences during an experimental paradigm, but the frequency was very low. Using the same paradigm, Vandenbroucke, Crombez, Harrar, et al. (2014) showed that the presence of chronic pain did not affect the frequency of somatosensory experiences. Derbyshire, Osborn, and Brown (2013) investigated the influences of prior pain experience and bodily ownership on the experience of vicarious sensations. They showed that the tendency to report vicarious experiences was enhanced when the type of observed pain (e.g., toothache) had been commonly experienced by the observer himor herself. Interestingly, previous studies have also demonstrated that the observation of pain facilitates the detection of tactile stimuli (Vandenbroucke, Crombez, Harrar, et al., 2014; Vandenbroucke, Crombez, Loeys, \& Goubert, 2014).

It is yet unclear whether the modulatory effects of observing pain on somatosensation are specific (or different) for pain or may equally be present when observing touch. Some studies have not investigated the experience in terms of behavioral somatosensory detection in response to the observation of painful stimuli, but rather have looked at somatosensory brain activity. Bufalari, Aprile, Avenanti, Di Russo, and Aglioti (2007) showed a reduction of somatosensory activity with respect to baseline when observing nonpainful tactile stimuli, in comparison with an increase when observing painful stimuli. Cheng, Yang, Lin, Lee, and Decety (2008) reported that observing both painful and nonpainful situations was associated with enhanced activation of somatosensory cortex, as compared with baseline. Martínez-Jauand et al. (2012) showed that the observation of both pain and touch video clips led to an enhancement of P50 amplitudes relative to viewing a hand without stimulation. Of particular relevance is the study of Valentini, Liang, Aglioti, and Iannetti (2012). These authors showed that viewing pain in another specifically modulates the neural activity in the onlooker's sensorimotor cortex, and that this modulation occurs only in the neural activity elicited by stimuli belonging to the nociceptive, rather than to another, sensory modality. There is evidence that observing touch improves tactile discrimination (Kennett et al., 2001) and that observing pain enhances detection accuracy (Vandenbroucke, Crombez, Harrar, et al., 2014; Vandenbroucke, Crombez, Loeys, \& Goubert, 2014). However, as yet no research has investigated whether there is a difference between observing touch versus pain in another. Some behavioral studies have focused on somatosensory modulations, but no study has directly compared the effects of observing pain and touch. Some studies have compared the effects of observations of human parts being touched versus the same parts merely being approached (Cardini et al., 2011; Serino, Pizzoferrato, \& Làdavas, 2008), of observing versus experiencing touch (Blakemore et al., 2005; Keysers et al., 2004), of observing touch to a person versus touch to an object (Blakemore et al., 2005; Cardini et al., 2011; Serino et al., 2008), of experiencing touch versus observing an object being touched (Keysers et al., 2004), and of observing pain versus observing an object being pricked or approached (Vandenbroucke, Crombez, Harrar, et al., 2014; Vandenbroucke, Crombez, Loeys, \& Goubert, 2014). The first aim of the present study was therefore to investigate whether the effects on vicarious experiences or the detection of somatosensory stimuli differ between the observation of touch versus pain in another.

A variable that may play a role in the production of vicarious experiences is perspective taking (Fitzgibbon, Giummarra, et al., 2010) - that is, whether one considers the observed pain or touch from a first-person or third-person (another's) perspective. It has been proposed that vicarious somatosensory experiences may be enhanced when a "self" perspective is adopted (Fitzgibbon, Giummarra, et al., 2010). No study has investigated this idea. However, studies have indicated that the adoption of a first-person perspective, by means of either an experimental paradigm or instructions or visual appearance facilitates/affects the detection of somatosensory stimuli (Loggia, Mogil, \& Bushnell, 2008; Serino, Giovagnoli, \& Làdavas, 2009; Serino et al., 2008). In the study of Loggia et al., similarity was manipulated by showing participants video interviews with an actor in which empathy for the actor was manipulated. At the end, participants saw the actor being exposed to stimuli similar to the ones they themselves were experiencing. Those in the high-similarity group rated the painful stimuli as more intense. Saxe, Jamal, and Powell (2006) showed that viewing body parts in firstperson perspective produced greater activation of the somatosensory cortex than did viewing the same parts in third-person perspective. Jackson, Meltzoff, and Decety (2006) found similar results for both imitating and viewing actions. Activations occurred in a wide area of the sensorimotor cortex and were greater for first-person perspective than for third-person perspective. At present, it is yet unclear whether taking a "self" perspective (vs. an "other" perspective) facilitates the experience of vicarious sensations. A second aim of the present study was to investigate the roles of perspective taking in vicarious somatosensory experiences and detection accuracy for subtle vibrotactile stimuli.

In a variant of the crossmodal congruency task, participants were presented with three categories of videos, depicting pain- 
related situations (left and right hands, of which one was being pricked), touch (left and right hands, of which one was touched by a cotton swab), and control situations (e.g., the same motor movement of the approaching hand as in the first and second categories, but without the painful/touching object). Participants received vibrotactile stimuli on the hand in the same spatial location (congruent trials) or in the opposite location (incongruent trials) as in the visual stimuli, or on both hands. Participants were instructed to report as quickly as possible the spatial location of the administered somatosensory stimuli. Also, trials in which no vibrotactile stimulation occurred were included, as well as trials in which both of the participant's hands were stimulated. To investigate the effect of perspective taking, videos were presented in a first-person and a third-person (i.e., videos were presented upside down) perspective. False alarms (erroneously reporting a somatosensory stimulus in the same spatial location as the visual cue) in response to videos showing pain or touch were labeled "vicarious somatosensory experiences."

First, we hypothesized that participants would report more vicarious experiences (false alarms) in response to the observation of pain than to the touch or control videos. Second, we expected that the observation of pain-related visual scenes would result in better detection accuracy for the vibrotactile stimuli, as compared with the touch and control videos. We furthermore expected to find a crossmodal congruency effect (CCE) in which more vibrotactile acuity would be observed when the visual and vibrotactile stimuli were congruent (i.e., presented in the same spatial location). We hypothesized that this CCE effect would be dependent on the type of visual information (pain-related vs. touch vs. control). More specifically, we expected a higher CCE when pain-related videos were shown than with the non-pain-related videos (touch and control). Third, we expected that pain-related videos presented in first-person perspective would facilitate detection accuracy and increase the report of vicarious experiences, as compared with pain-related videos presented in thirdperson perspective. In addition, we also explored the presence of neglect errors (i.e., only reporting the site congruent to the visual information when both hands were stimulated) during the observation of each category of video and perspective. As in previous studies (Vandenbroucke, Crombez, Harrar, et al., 2014; Vandenbroucke, Crombez, Loeys, \& Goubert, 2014; Vandenbroucke et al., 2013), we investigated the putative roles of some individual difference variables in vicarious experiences. In the model of Fitzgibbon, Giummarra, et al. (2010), it was suggested that individual differences in empathy and hypervigilance to pain would lead to more vicarious experiences. For that reason, we assessed both variables through self-report questionnaires and explored their roles in vicarious experiences and the detection of vibrotactile stimuli.

\section{Method}

\section{Participants}

Undergraduate psychology students $(n=57)$ were recruited by means of an online system to which they could subscribe for experiments. They were paid $€ 10$ for participation. In all, $75 \%$ of the participants were female, and $79 \%$ were righthanded by self-report. All were Caucasian. The average age of the participants was 23.68 years $(S D=4.62)$, and they rated their general health on average as "Very good." Sixty-three percent of the participants reported having experienced pain during the last six months (average of 27.6 days in 6 months). Fourteen of the participants reported pain at present (score $>0$ on a Likert scale, where 0 indicated no pain and 10 the worst pain ever; assessment before the experiment), but the average intensity was low $(M=2.64, S D=1.78)$. All participants gave informed consent and were informed that they were free to terminate the experiment at any time. None made use of this possibility. Ethical approval was obtained from the Ethics Committee of the Faculty of Psychology and Educational Sciences.

\section{Self report measures}

Vigilance to pain was assessed by the Dutch version of the Pain Vigilance and Awareness Questionnaire (PVAQ; McCracken, 1997; Roelofs, Peters, Muris, \& Vlaeyen, 2002). This questionnaire consists of 16 items assessing awareness, consciousness, and vigilance to pain on a 6-point scale $(0=$ never, $5=$ always $)$. Higher scores on the PVAQ are indicative of greater pain-related vigilance and awareness. The questionnaire can be used with both clinical (McCracken, 1997; Roelofs, Peters, McCracken, \& Vlaeyen, 2003) and nonclinical (McWilliams \& Asmundson, 2001; Roelofs et al., 2002) samples. The Dutch version of the PVAQ is reliable and valid (Roelofs et al., 2003; Roelofs et al., 2002). Cronbach's alpha in the present study was .91 .

Empathic disposition was assessed by means of the Dutch version of the Interpersonal Reactivity Index (IRI; Davis, 1983; De Corte et al., 2007). The questionnaire contains 28 items and consists of four subscales: Perspective Taking (i.e., cognitively taking the perspective of another; e.g., "I sometimes try to understand my friends better by imagining how things look from their perspective.”), Fantasy (i.e., emotional identification with characters in books, movies, etc.; e.g., "When I watch a good movie, I can very easily put myself in the place of a leading character."), Empathic Concern (i.e., feeling emotional concern for others; e.g., "I am often quite touched by things that I see happen."), and Personal Distress (i.e., negative feelings in response to the distress of others; e.g., "When I see someone who badly needs help in an emergency, I go to pieces."). Each item is answered on a scale 
ranging from 1 (does not describe me very well) to 5 (describes me very well). This questionnaire has been shown to be reliable and valid (Davis, 1983; De Corte et al., 2007). Cronbach's alphas in the present study were .78 (Fantasy scale), .80 (Personal Distress), .64 (Perspective Taking), and .60 (Empathic Concern). Perspective taking and empathic concern were omitted from the analyses because of the low reliability scores.

Vicarious pain experiences during daily life were measured by means of four items adapted from Banissy, Kadosh, Maus, Walsh, and Ward (2009). Participants were asked to indicate on an 11-point scale $(0-10$, totally disagree-totally agree $)$ the extent to which they agreed with the following questions: "Do you feel pain in your own body when you see someone accidently bump against the corner of the table?," "Do you have the feeling experiencing pain when you observe another person in pain?," "Do you feel bodily pain when you observe another person in pain?," and "Do you feel a physical sensation (e.g., tingling, stabbing) when you observe another person in pain." We have used this adapted instrument in previous studies (Vandenbroucke, Crombez, Harrar, et al., 2014; Vandenbroucke et al., 2013). In the present study, Cronbach's alpha was .87.

\section{Procedure}

\section{Behavioral paradigm}

Preparation phase First, for each participant, the threshold intensity level for the vibrotactile stimuli was individually determined prior to the experiment. Vibrotactile stimuli $(50 \mathrm{~Hz}, 50 \mathrm{~ms})$ were delivered by two resonant-type tactors (C-2 tactor, Engineering Acoustics, Inc.) consisting of a housing that was $3.05 \mathrm{~cm}$ in diameter and $0.79 \mathrm{~cm}$ high, with a skin contactor that was $0.76 \mathrm{~cm}$ in diameter. The vibrotactile stimuli were delivered on the skin between the thumb and index finger. All stimulus characteristics (amplitude, duration, and frequency) were entered through a self-developed software program that was used to control the tactors. Four different series of 20 stimuli/trials (two series for each hand) were randomly administered (80 stimuli/trials in total). First, a visual stimulus " $\mathrm{X}$ " was presented, combined with a somatosensory stimulus on the left or the right hand. Participants were instructed to report whether they felt a somatosensory stimulus ("yes" or "no"), which was coded by the experimenter, by pressing the corresponding response button (see Vandenbroucke, Crombez, Harrar, et al., 2014; Vandenbroucke, Crombez, Loeys, \& Goubert, 2014). Each series started at $0.068 \mathrm{~W}$, and this intensity decreased by $0.0002 \mathrm{~W}$ within each series when participants reported feeling a stimulus, and increased by $0.0002 \mathrm{~W}$ when no sensation was reported. After 80 trials, this resulted in a threshold intensity for each hand that was based on the mean intensity of the last stimuli (20th) of the two series for that particular hand. From this threshold intensity (threshold left hand: $M=$ $0.033 \mathrm{~W}, S D=0.008$, range: $0.002-0.174 \mathrm{~W}$; threshold right hand: $M=0.038 \mathrm{~W}, S D=0.006$, range: $0.003-0.163 \mathrm{~W}), 1 / 8$ was added to the threshold (above threshold), resulting in four different intensities (threshold and above threshold, one for each hand). Several intensities were implied, in order not to habituate participants to the intensity as well as to enhance the chance to make vicarious errors.

Second, participants were informed that during the experiment they would feel subtle stimuli, varying in intensity and length, on their left, on their right, or on both hands. Participants were instructed that different videos would be presented that they would need to watch attentively, that when a somatosensory stimulus was administered on both hands, the intensity could vary across hands, and that trials without any stimulus would also be included. In reality, only two fixed, predetermined intensities were applied for a fixed duration (threshold intensity and threshold intensity $+1 / 8$ ).

Experimental phase The visual stimuli consisted of three categories of videos (pain, control, and touch) with a duration of 3,000 ms. The first, "pain" category included a scene depicting a left and a right hand, with one of the two hands being pricked by a syringe (2,000 ms after video onset). The second category depicted a touch scene: A left and a right hand were presented, and one of these hands was touched by a cotton swab (2,000 ms after video onset). The third, "control" category included a scene depicting a left and a right hand, in which one hand was approached by a hand that was not holding an object (same movement of the approaching hand as in the first and second categories of videos). Videos were presented by the Inquisit Millisecond software (Inquisit, 2002) on a Dell screen with a 19-in. CRT monitor. The computer screen was placed in front of the participants at approximately $22^{\circ}$, and participants' hands were placed underneath the screen. The left hand was placed at the left and the right at the right under the screen, to make the perspective-taking manipulation more salient.

Each trial began with a fixation cross (1,000-ms duration) presented in the middle of the computer screen. Next, one of the videos was presented. In $75 \%$ of the trials, a vibrotactile stimulus was delivered $2,450 \mathrm{~ms}$ after video onset to the left hand, to the right hand, or to both hands of the participant. In line with Banissy and Ward (2007), the somatosensory stimulus was administered with a delay of $450 \mathrm{~ms}$ after the visual stimulus of penetration of the needle or touch of the cotton swab (see Vandenbroucke, Crombez, Harrar, et al., 2014; Vandenbroucke, Crombez, Loeys, \& Goubert, 2014). For the control videos, the somatosensory stimulus was administered with a delay of $450 \mathrm{~ms}$ after the approaching hand was closest to the resting hand (same time frame as in the other video categories). This resulted in the following trial 
types: congruent trials, incongruent trials, and trials in which no somatosensory stimuli were administered or in which both of the participant's hands received somatosensory stimuli. In congruent trials, the somatosensory and visual stimuli were presented at the same spatial location (e.g., on the right). In incongruent trials, the somatosensory and visual stimuli were presented in the opposite spatial locations (e.g., on the left and right). The experiment started with eight practice trials. The actual experimental phase consisted of five blocks of 96 trials, resulting in a total of 480 trials. Of these, 120 were congruent trials, 120 were incongruent trials, 120 were trials without sensory stimuli, and 120 were trials with somatosensory stimuli to both hands. These three categories of videos were presented in equal numbers in first-person perspective (240 trials; i.e., presented in the same orientation as the hands of the participant) and in third-person perspective (240 trials; i.e., the same videos were turned upside down) (see Fig. 1). The different categories, locations of visual cues (touch, pain, and control), congruencies (congruent, incongruent, both hands stimulated, and both hands not stimulated), and perspectives (first vs. third person) were counterbalanced across videos. The order of trial types was randomized within each block, and the somatosensory stimuli were equally distributed within and over blocks, types of intensity (threshold and above threshold), and perspectives (first vs. third person).

An overview of all trial types is presented in Table 1. During each trial, participants were requested to report whether a somatosensory experience was felt by reporting as quickly as possible "YES," and to discriminate the spatial location of the somatosensory stimuli by reporting "left," "right," or "both" (see Fig. 2). After the video had ended and 2,000 ms had been elapsed, the Dutch word for "next" was presented on the screen. Then the experimenter coded the response by pressing the corresponding response button (left, right, both, or no response; see Fig. 1). In this way, the time to respond was equal for every participant. The experiment took approximately $1 \mathrm{~h}$.

Postexperiment phase After the experiment, participants were requested to fill out self-report scales measuring hypervigilance for pain (PVAQ), empathic disposition (IRI), and the items measuring vicarious pain experiences during daily life, which took approximately $15 \mathrm{~min}$.

\section{Statistical analysis}

\section{False alarms}

The number of false alarms was calculated from the incongruent trials and from trials without any somatosensory stimuli when a somatosensory stimulus was erroneously reported in the same spatial location as the visual cue (i.e., site of the touch/prick or approaching movement). These false alarms were labeled "vicarious somatosensory experiences" when the visual stimulus was of pain or touch. To test whether the category of video predicted the number of false alarms, generalized linear mixed models for the count data were applied. The use of linear models is considered less appropriate (Vives, Losilla, \& Rodrigo, 2006) when the frequency of responses has a skewed distribution that violates the normality assumption. Poisson regression is the basic model to analyze count data, but the variance of counts is often larger than the mean (overdispersion). The negative binomial (NB) regression, a Poisson regression with an overdispersion, might therefore fit the data better (see, e.g., Gardner, Mulvey, \& Shaw, 1995). Because count data may additionally exhibit a lot of
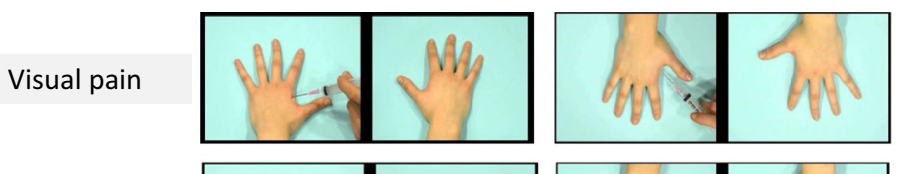

Visual touch
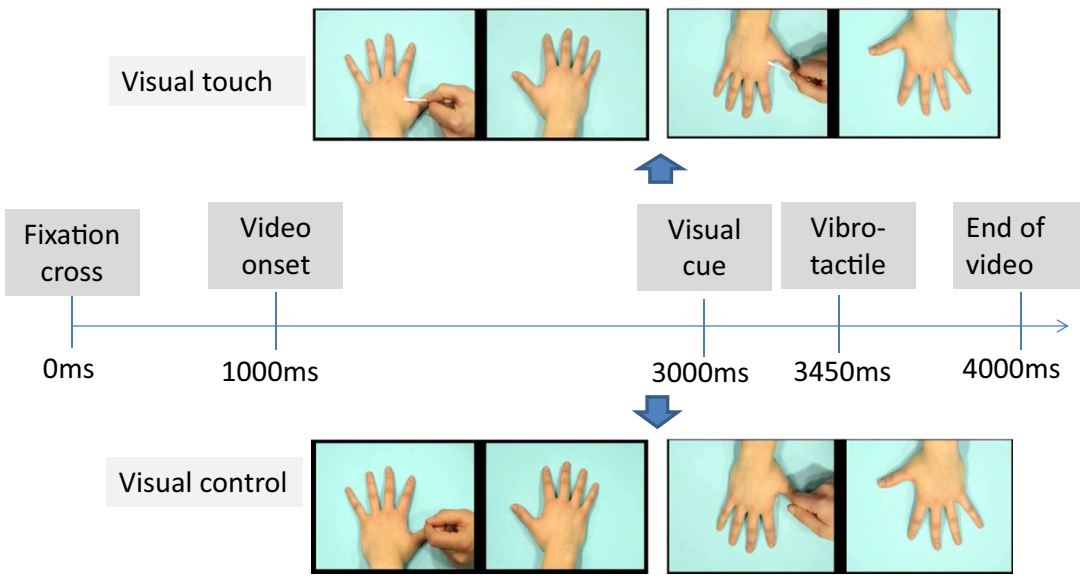

ᄂ

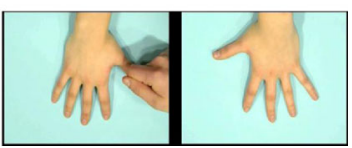

Fig. 1 Time line of a trial including vibrotactile stimulation 


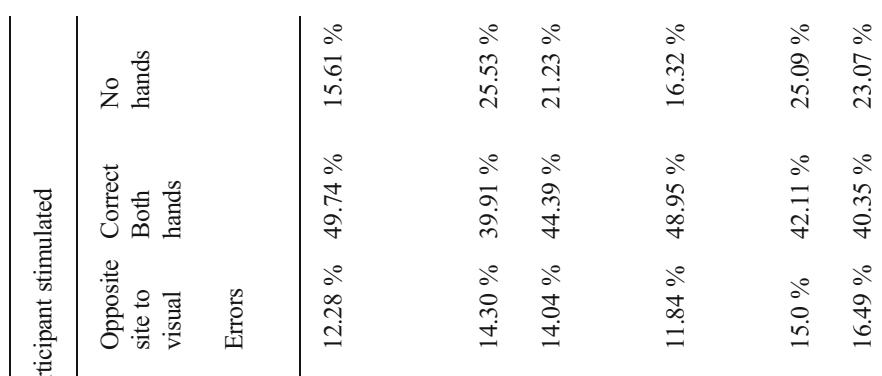

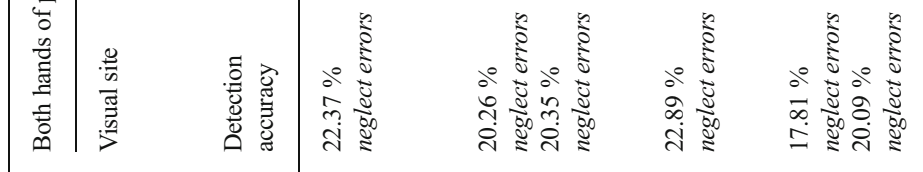

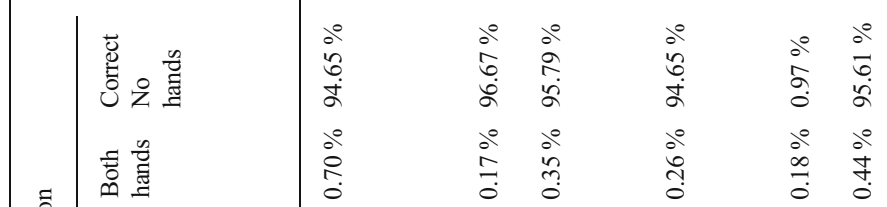

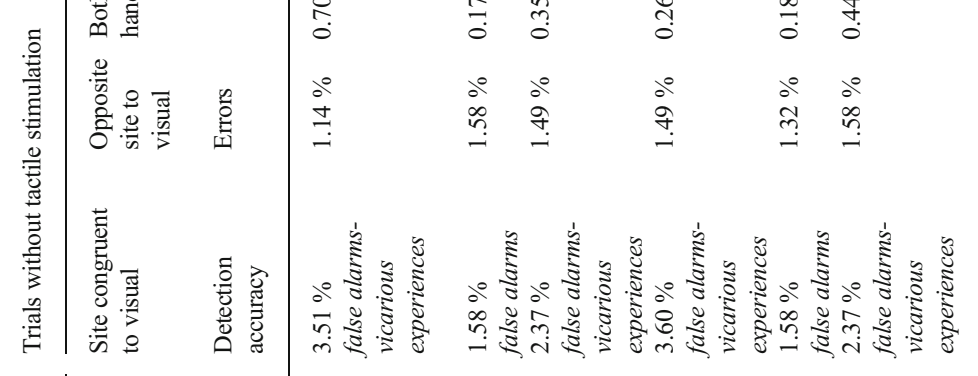

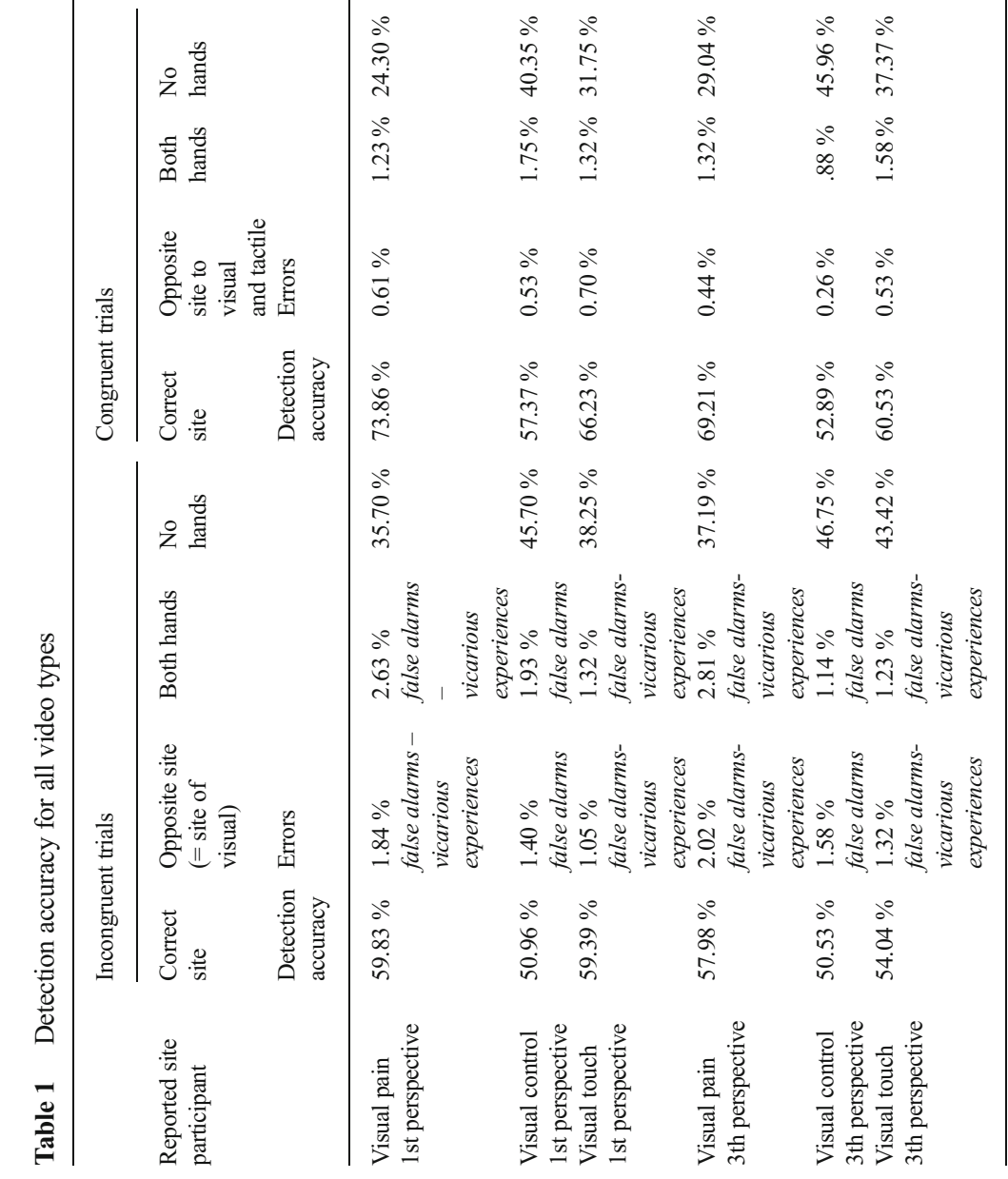



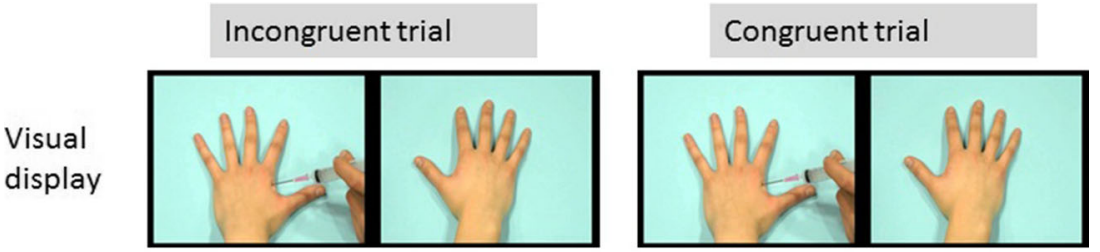

Participant
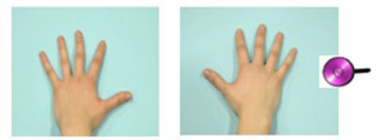

Correct response $=$ right vicarious error $=$ both or left

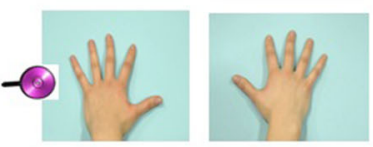

Correct response $=$ left
Other error $=$ right, both or
none

Fig. 2 Example of possible trials

zero counts, zero-inflated extensions of both models, called zero-inflated Poisson and zero-inflated NB models, have been developed (see Karazsia \& van Dulmen, 2010; Loeys, Moerkerke, De Smet, \& Buysse, 2012). Deviance tests and the Vuong test were used to select the best-fitting count distribution for the dependent variable. After the best-fitting count model was chosen, a first model with "video category" as a predictor was added. In a further exploration of the data, hypervigilance for pain, dispositional empathy, and their interactions with video category were added in separate models to test whether they had a moderating role. Dummy coding was used for the categorical variables. Regression coefficients were exponentiated $(\mathrm{eB})$ and called rate ratios (RRs). In percentages $-100 \times(\mathrm{eB}-1)-\mathrm{RRs}$ reflect the percentage decrease $(R R<1)$ or increase $(R R>1)$ in the expected frequency of false alarms for each one-unit increase in the continuous predictor. In a second series of analyses, the above-mentioned analyses were repeated with "perspective" (first vs. third person) as a predictor. In a third model, both video category and perspective were added as predictors. $\mathrm{R}$ (version 2.15.1) was used to fit the count models.

\section{Detection accuracy}

To investigate whether type of video category and type of perspective taking modulated detection of the vibrotactile stimuli, the proportions of correct responses (left vs. right) for congruent and incongruent trials for each category of visual information were calculated (pain-related, touch, and control). A 3 (video category: pain-related vs. touch vs. control $) \times 2$ (congruency: congruent vs. incongruent $) \times 2$ (perspective: first vs. third person) repeated measures analysis of variance (ANOVA) was performed, with congruency, video category, and perspective entered as withinsubjects variables. In a further exploration, hypervigilance for pain and dispositional empathy were added as the covariates in separate models, to test whether they had a moderating role. Repeated measures ANOVAs were conducted with alpha $<.05$, using the SPSS statistical software, version 21.0 for Windows.

\section{Neglect errors}

The number of neglect errors was calculated on the basis of those trials on which both hands were stimulated, defined as reporting only the site congruent to the visual information (i.e., the site of the touch/prick or approaching movement) and missing the actual vibrotactile stimuli on both hands. Generalized linear mixed models for the count data were applied again to test whether the number of neglect errors was dependent on the type of video and perspective. After the bestfitting count model was chosen, a first model with "type of video" as a predictor was added. In a further exploration, hypervigilance for pain, dispositional empathy, and their interactions with type of video were added in separate models to test whether they had a moderating role. In a second series of analyses, "perspective" (first vs. third person) was added as predictor. In a third model, both video category and perspective were added as predictors. R (version 2.15.1) was used to fit the count models.

\section{Results}

\section{Descriptives}

Mean scores, standard deviations, and correlations are presented in Table 2. Spearman correlations were computed for the nonnormally distributed variables (KolmogorovSmirnoff, $p<.05$ ). Without taking type of video into account, false alarms were made on $2.94 \%$ of the incongruent trials and the trials without vibrotactile stimuli (402 false alarms 
Table 2 Pearson/Spearman correlations, mean scores, and standard deviations of all measures

\begin{tabular}{|c|c|c|c|c|c|c|c|c|}
\hline & $M(S D)$ & 2. & 3. & 4. & 5. & 6. & 7. & 8. \\
\hline 1. Vicarious somatosensory errors (pain videos, first person) & $1.60(2.69)$ & $47^{* *}$ & $.40^{* *}$ & $.36^{* *}$ & .24 & .07 & .14 & .11 \\
\hline 2. Vicarious somatosensory errors (pain videos, third person) & $1.68(3.00)$ & - & $.30^{*}$ & .11 & .07 & .03 & .03 & .21 \\
\hline 3. Neglect errors (pain videos, first person) & $4.47(3.06)$ & & - & $.54^{* *}$ & .09 & .03 & .02 & .23 \\
\hline 4. Neglect errors (pain videos, third person) & $4.58(2.32)$ & & & - & .15 & .14 & .02 & -.05 \\
\hline 5. Hypervigilance for pain (PVAQ) & $33.95(13.52)$ & & & & - & .22 & .10 & -.01 \\
\hline 6. Personal distress (IRI) & $12.97(4.62)$ & & & & & - & .04 & .05 \\
\hline 7. Fantasy (IRI) & $19.11(4.73)$ & & & & & & - & .04 \\
\hline 8. Vicarious pain in daily life (sum score of four items) & $15.09(9.18)$ & & & & & & & - \\
\hline
\end{tabular}

$\mathrm{PVAQ}=$ Pain Vigilance and Awareness Questionnaire, IRI $=$ Interpersonal Reactivity Index. ${ }^{*} p<.05 ;{ }^{* *} p<.01$

from a total of 13,680 trials). Vicarious somatosensory errors in response to the observation of pain-related scenes were made on $4.10 \%$ of the incongruent and no-stimulation trials (187 vicarious somatosensory errors from a total of 4,560 trials). Of these vicarious somatosensory errors, $48.66 \%$ occurred when the pain-related video was in first-person perspective (91 from a total of 187 vicarious somatosensory errors). Vicarious somatosensory errors in response to observation of the touch scenes were made on $2.41 \%$ of the incongruent and no-stimulation trials (110 vicarious somatosensory errors from a total of 4,560 trials). Of these vicarious somatosensory errors, $49.09 \%$ occurred when the touch video was in first-person perspective (54 from a total of 110 vicarious somatosensory errors). Neglect errors were made on $20.63 \%$ of the trials in which both hands were stimulated (1,411 neglect errors from a total of 6,840 trials). Such errors were made on $22.63 \%$ of all trials with pain-related videos (516 neglect errors from a total of 2,280 trials). Of these neglect errors, $255(49.42 \%)$ occurred when the pain-related video was shown in first-person perspective.

\section{False alarms and vicarious experiences}

The NB model was found to be the best-fitting count model. In a first step, video category was added as a predictor. The results showed that the number of false alarms was dependent on the type of video presented. The observation of painrelated videos resulted in an $81 \%$ increase in false alarms, as compared with control videos $(\mathrm{RR}=1.81 ; p<.001)$. The observation of pain-related videos resulted in a $70 \%$ increase in false alarms or vicarious experiences relative to touch videos $(\mathrm{RR}=1.81 ; p<.001)$. No significant difference was found between touch videos and control videos regarding the number of false alarms made $(p=.70)$. In order to explore the role of individual differences in PVAQ and the IRI, several additional models were run with PVAQ or IRI as an additional predictor, to explore their modulating roles. No interactions were found between type of video and PVAQ (all $p \mathrm{~s}>.18$ ), Personal Distress (all $p \mathrm{~s}>.28$ ), or Fantasy scale (all $p \mathrm{~s}>.41$ ).

In a separate model, perspective was added as a predictor. The results showed that the number of false alarms was independent of the type of perspective $(p=.89)$. In a third model, both type of video and perspective were added as predictors. No interaction occurred between video category and perspective (all $p s>.64)$.

\section{Detection accuracy}

A 2 (congruency: congruent vs. incongruent) $\times 2$ (type of perspective: first vs. third person) $\times 3$ (type of video: pain vs. touch vs. control) repeated measures ANOVA showed a main effect of type of video $[F(2,112)=41.49, p<.001]$. Overall, pain-related videos resulted in better detection of vibrotactile stimuli than did control videos $[t(56)=$ 7.99, $p<.0001$, Cohen's $d=0.68,95 \%$ CI: $0.49,0.86]$ or touch videos $[t(56)=4.29, p<.0001$, Cohen's $d=0.27,95 \%$ CI: $0.15,0.39]$. Detection accuracy while observing touch videos was significantly higher than while observing control videos $[t(56)=-5.48, p<.0001$, Cohen's $d=0.37,95 \% \mathrm{CI}$ : $0.23,0.51]$. Also, a main effect of congruency occurred $[F(1,56)=64.23, p<.0001$, Cohen's $d=0.43,95 \% \mathrm{CI}$ : $0.32,0.54]$, indicating higher detection accuracy in congruent than in incongruent trials.

An interaction was found between congruency and type of video: The CCE was dependent on the type of video presented $[F(2,112)=7.42, p=.001]$. A pairedsamples $t$ test showed that the CCE was present for each type of video [pain video: $t(56)=-6.66, p<.0001$, Cohen's $d=-0.63,95 \% \mathrm{CI}:-0.84,-0.43$; control video: $t(56)=-3.11$, $p=.003$, Cohen's $d=-0.23,95 \% \mathrm{CI}:-0.38,-0.08$; touch video: $t(56)=-4.48, p<.0001$, Cohen's $d=-0.32,95 \%$ CI: $0.47,-0.18]$. The congruency effect was, however, significantly larger for pain videos than for control videos $[t(56)=$ 3.56, $p=.001$, Cohen's $d=0.65,95 \% \mathrm{CI}: 0.26,1.05]$ or touch videos $[t(56)=2.66, p=.01$, Cohen's $d=0.46,95 \% \mathrm{CI}$ : 
$0.10,0.82]$. The congruency effects were not significantly different for touch videos and control videos $[t(56)=-1.10$, $p=.28$, Cohen's $d=-0.21,95 \%$ CI: $-0.58,0.16$ ] (see Fig. 3). Also, a main effect of perspective was found $[F(1,56)=$ $24.59, p<.0001$, Cohen's $d=-0.20,95 \%$ CI: -0.28 , $0.12]$, indicating that observing videos in first-person perspective resulted in better detection than did observing videos shown in third-person perspective. No interactions were found between type of perspective and type of video category $[F(2,112)=1.75, p=.18]$, between type of perspective and congruency $[F(1,56)=2.60, p=.11]$, and between type of perspective, type of video category, and congruency $[F(2,112)=0.55, p=.58]$. The centered PVAQ and IRI subscales were entered separately as covariates. No main effects were found for PVAQ, $F(1,55)=0.20, p=.66$, Fantasy scale, $F(1,55)=0.85, p=.36$, and Personal Distress, $F(1,55)=0.00, p=.99$.

\section{Neglect errors}

The NB model was found to be the best-fitting count model. In a first step, type of video was added as a predictor. The results showed that the number of neglect errors during the observation of pain-related stimuli was dependent on video category. The observation of pain-related videos resulted in a $19 \%$ increase in neglect errors, as compared with control videos $(\mathrm{RR}=1.19 ; p=.008)$. No difference was found between control and touch videos $(p=.37)$ or between pain and touch $(p=.08)$. In order to explore the role of individual differences in PVAQ and the IRI scores, several additional models were run with PVAQ or IRI as an additional predictor and in interaction with group, to explore its modulating role.
No interactions were found between video category and PVAQ (all $p \mathrm{~s}>.26$ ) and Fantasy (all $p \mathrm{~s}>.30$ ). The effects of personal distress on the number of neglect errors were significantly different for touch and pain-related videos $(p=.01)$ : The number of neglect errors decreased for every one-unit increase in personal distress by $1 \%(\mathrm{RR}=.99)$ when touch videos were presented, and increased by $2 \%$ when painrelated videos were presented $(R R=1.02)$. Second, in a separate model, type of perspective was added as a predictor. The results showed that the number of neglect errors was independent of type of perspective $(p=.51)$. In a third model, both type of video and perspective were added as predictors. No interactions occurred between video category and perspective (all $p \mathrm{~s}>.24)$.

\section{Discussion}

This study had two objectives. First, we investigated whether the observation of touch and pain differentially facilitated the report of vicarious experiences and the detection of subtle somatosensory stimuli during an experimental paradigm. Second, we tested whether perspective taking (first vs. third person) influenced these outcomes. We also explored the effects of some potential moderators proposed by Fitzgibbon et al. (2012; Fitzgibbon, Giummarra, et al., 2010) - that is, dispositional empathy and hypervigilance to pain. Participants were presented with three categories of videos, showing pain-related scenes (left and right hand, one of which was being pricked), touch scenes (left and right hand, one of which was touched by a cotton swab), and control situations (e.g., the same approaching movement of the hand

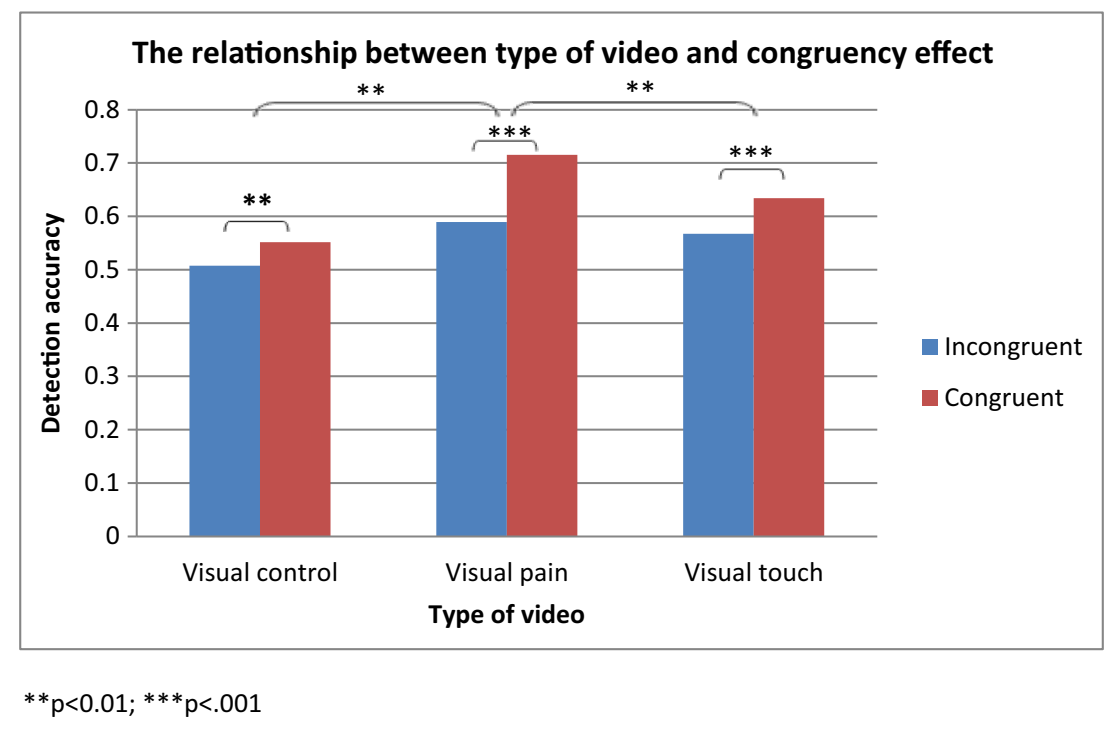

Fig. 3 Relationship between type of video and congruency. ${ }^{* *} p<.01 ;{ }^{* * *} p<.001$ 
as in the other categories, but without holding any object). Videos were presented in first-person (self) and third-person (other) perspectives; in the latter case, the videos were turned upside down. Participants occasionally received vibrotactile stimuli themselves at the same spatial location (congruent trials) or the opposite location (incongruent trials) as the visual cue (touch/prick or approaching movement). Participants were instructed to report as rapidly as possible the spatial location of the administered somatosensory stimuli.

The results can be readily summarized. First, observing pain in another increased the number of vicarious experiences and improved the accuracy of detecting somatosensory stimuli. Second, we did not observe an increase of vicarious experiences when pain or touch was observed in the first-person perspective, as compared with the third-person perspective. Nevertheless, observing pain and touch in the firstperson perspective did improve detection accuracy of the somatosensory stimuli. Third, no moderating role was found for observers' characteristics, such as hypervigilance and dispositional empathy. Our results corroborate previous findings, since they show that vicarious experiences are not frequently reported but can be measured by means of an experimental paradigm (Vandenbroucke, Crombez, Harrar, et al., 2014; Vandenbroucke, Crombez, Loeys, \& Goubert, 2014; Vandenbroucke et al., 2013). Of particular relevance to this study was whether the effects are specific to observing pain.

Our primary finding, that participants reported more vicarious somatosensory experiences when pain-related videos were shown, relative to control and touch videos, indicates that vicarious experiences while observing pain are not simply due to the observation of a hand being approached or touched. Rather, vicarious experiences become more frequent when observing pain-related situations, in comparison with touch situations. No difference was obtained regarding the number of vicarious somatosensory experiences while observing touch as compared with control videos. Mirams, Poliakoff, Brown, and Lloyd (2010) found that merely viewing a hand increases the number of false alarms, as compared to not viewing a hand. In our study, false alarms may have also been facilitated in the control condition, since there was no condition in which no hand was seen. Also in our control videos, human features such as a hand were still present.

Detection accuracy was also affected by the type of video presented. Participants were better at detecting the vibrotactile stimuli while observing pain-related situations, as compared with both touch and control videos. Observing touch resulted in better detection than did observing control videos. In line with our hypotheses, spatially congruent visual information resulted in better detection than did incongruent trials. As expected, this congruency effect was present when touch and control videos were shown, although to a lesser extent than during the presentation of pain-related videos. These effects are consistent with previous research comparing the effects of pain-related videos and control videos on somatosensation (Vandenbroucke, Crombez, Harrar, et al., 2014). The increased detection accuracy while observing touch in this study is congruent with previous studies demonstrating that observing nonpainful touch may facilitate somatosensory experiences (e.g., Cardini et al., 2013; Serino et al., 2008). Common pathways exist in experiencing touch and pain, such as multimodal neurons that respond to both nociceptive and nonnociceptive inputs (Mouraux, Diukova, Lee, Wise, \& Iannetti, 2011). Besides these common pathways for experiencing touch and pain, our results suggest that the different video categories (pain, touch, and control) modulate somatosensation differently. This difference is consistent with the existence of different neurophysiological mechanisms of viewing painful and nonpainful bodily sensations in others (Bufalari et al., 2007). One possible explanation for these results is that participants may have been more aroused when viewing the pain videos than when viewing the control and touch videos. Because pain captures attention and may induce threat, it may have been more arousing (in a way this is an inherent feature of pain stimuli).

Another important mechanism is the involvement of attentional processes. Attention may enhance the sensory processing of somatic information when observing bodily experiences in others, irrespective of whether or not they are painful. Martínez-Jauand and colleagues (2012) showed enhanced P50 amplitudes for the sight of bodily sensations, irrespective of whether participants were observing either a painful or a nonpainful bodily sensation. This suggests that images of body parts interacting with an object are able to capture participants' attention to a larger extent than do images of a body not receiving stimulation. Further research may focus upon possible explanatory variables for our findings - for example, the mediating roles of arousal and attentional processes. Serino et al. (2008) demonstrated enhanced detection of subthreshold tactile stimuli on observers' faces when they saw a face being touched by hands rather than a face merely being approached by hands. This effect was not found for touch on a nonbodily stimulus - namely, a picture of a house. One explanation could be that because of presentation of the inanimate house object, perception was already diminished, independent of an approaching or touching condition. Beck, Bertini, Scarpazza, and Làdavas (2013) showed no modulation of detection while observing touch to monkey faces expressing different facial expressions (fearful, happy, or neutral), which does occur when presenting human faces, illustrating that the simple presentation of human features may influence detection accuracy. A particular strength in the present study is, therefore, that even in the nonpainful videos in this study, human features were still present. The effects in our study are unlikely to be due to the mere observation of the human body, since human hands were present in all video categories. 
Also, the type of perspective was important, regarding detection accuracy: Participants were better at detecting the vibrotactile stimuli when videos were presented in firstperson rather than third-person perspective. Contrary to our expectations, the role of perspective was not dependent on the type of visual information, suggesting that any hand observed in first-person perspective facilitated detection, as compared with third-person perspective. This effect of perspective taking on detection accuracy is in line with research done by Serino et al. (2009). These researchers showed that vision facilitated tactile perception mostly when self-other similarity was high (e.g., by manipulating the visual appearance and political opinions between an observer and the observed person). The number of vicarious somatosensory experiences was independent of type of perspective. This suggests that perspective taking may be important but is largely dependent on the outcome (vicarious somatosensory experiences vs. general accuracy in detecting somatosensory stimuli).

In general, the effects of observing pain and touch and the role of perspective taking were stronger with regard to detection accuracy than to the sensation of vicarious experiences. This is in line with the view that vicarious experiences while observing touch or pain are a more extreme variant of the modulation of somatosensory detection in a minority of people (Vandenbroucke, Crombez, Loeys, \& Goubert, 2014; Vandenbroucke et al., 2013): Percentages range from 1.6\% for vicarious touch (Banissy et al., 2009), to $16.20 \%$ for vicarious pain in amputees (Fitzgibbon et al., 2010a), to $6.61 \%$ (Vandenbroucke et al., 2013, Study 1), $22.90 \%$ (Vandenbroucke et al., 2013, Study 2), and $30 \%$ for vicarious pain in a general population (Osborn \& Derbyshire, 2010). The variability is probably dependent on the criteria used for categorizing individuals as vicarious pain responders. Stability has been observed at a group level of vicarious pain responders reporting vicarious pain in daily life, but some variation may occur at the individual level (Vandenbroucke, Crombez, Loeys, \& Goubert, 2014). The study described in this article has made unique contributions to the literature beyond those of previous studies in our lab (Vandenbroucke, Crombez, Harrar, et al., 2014; Vandenbroucke, Crombez, Loeys, \& Goubert, 2014; Vandenbroucke et al., 2013), since in it we made a direct comparison between observing pain, touch, and control videos and reports of vicarious experiences and somatosensory modulation.

Regarding the number of neglect errors, observing painrelated scenes resulted in a higher number of neglect errors than did observing control scenes, but no differences were found between the observation of pain-related versus touch scenes. Personal distress in the context of empathy influenced the numbers of neglect errors differently for touch and painrelated videos. Fewer neglect errors were made when more personal distressed participants observed touch videos, versus more such errors when distressed observers watched pain- related videos. One possible explanation may be that when observing pain-related information in combination with the experience of personal distress, people become more attentive to the site of the pain-related information, resulting in more neglect errors.

Some limitations deserve further consideration, which point to directions for future research. First, we included video clips showing hands being pricked. It may well be that these videos represented pain experiences of lower intensity than did the images and movies (e.g., broken leg) used in the study of Osborn and Derbyshire (2010). Further studies should investigate whether effects differ as a function of pain intensity. It may well be that high-intensity scenes may lead to more vicarious experiences.

Second, we designed our videos to be as similar as possible, in terms of visual features as well as of the represented actions. For that reason, the control videos consisted of a hand approaching another hand without holding an object. Morrison, Tipper, Fenton-Adams, and Bach (2013) showed that separate somatosensory regions responded more strongly when the observed action targeted noxious rather than neutral objects, irrespective of the action carried out with them. This suggests an encoding of tactile object properties independent of action properties. Besides the differential influence of the presence of the absence of an approaching object, the type of object could also have played a role in our study (e.g., cotton swab vs. needle), in that a needle could have been more salient.

Third, our video clips were shown in peripersonal space, since the computer screen was placed just above participants' hands. Visual cues presented near the hands may facilitate the detection of stimuli delivered on these hands, as compared with visual cues farther away (see De Paepe, Crombez, Spence, \& Legrain, 2014). The fact that our video clips were presented close to the body may have overruled some hypothesized effects of perspective taking.

Fourth, in contrast to previous studies by Vandenbroucke et al. (2013; Vandenbroucke, Crombez, Loeys, \& Goubert, 2014), undergraduate students were used as participants. Future research may include participants who report vicarious experiences in daily life (vicarious pain responders) and controls in order to investigate the effects of observing touch and pain on somatosensation and vicarious experiences and their potentially different impacts on both groups.

Finally, future research may attempt to manipulate activity in the brain regions presumed to play a critical role in perspective taking. For example the temperoparietal junction (TPJ) is linked to self-other representations, including perspective taking (e.g., Aichhorn, Perner, Kronbichler, Staffen, \& Ladurner, 2006), agency discrimination (e.g., Farrer \& Frith, 2002), and empathy (e.g., Völlm et al., 2006). To get further insight into the role self-other representations in somatosensation, it would be interesting to manipulate the activity of TPJ and 
investigate its role in somatosensation while observing touch, pain, and control videos in an experimental setup like the one described in our study.

Author note This research was supported by the Special Research Fund (BOF), Ghent University, Grant Number 01D38311. The authors declare no conflicts of interest.

\section{References}

Aichhorn, M., Perner, J., Kronbichler, M., Staffen, W., \& Ladurner, G. (2006). Do visual perspective tasks need theory of mind? NeuroImage, 30, 1059-1068. doi:10.1016/j.neuroimage.2005.10.026

Banissy, M. J., Kadosh, R. C., Maus, G. W., Walsh, V., \& Ward, J. (2009). Prevalence, characteristics and a neurocognitive model of mirrortouch synaesthesia. Experimental Brain Research, 198, 261-272.

Banissy, M., \& Ward, J. (2007). Mirror-touch synesthesia is linked with empathy. Nature Neuroscience, 10, 815-816.

Beck, B., Bertini, C., Scarpazza, C., \& Làdavas, E. (2013). Observed touch on a non-human face is not remapped onto the human observer's own face. PLoS ONE, 8(e73681), 1-5. doi:10.1371/journal. pone. 0073681

Blakemore, S. J., Bristow, D., Bird, G., Frith, C., \& Ward, J. (2005). Somatosensory activations during the observation of touch and a case of vision-touch synaesthesia. Brain, 128, 1571-1583.

Bufalari, I., Aprile, T., Avenanti, A., Di Russo, F., \& Aglioti, S. M. (2007). Empathy for pain and touch in the human somatosensory cortex. Cerebral Cortex, 17, 2553-2561. doi:10.1093/cercor/bhl161

Cardini, F., Costantini, M., Galati, G., Romani, G. L., Làdavas, E., \& Serino, A. (2011). Viewing one's own face being touched modulates tactile perception: An fMRI study. Journal of Cognitive Neuroscience, 23, 503-513. doi:10.1162/jocn.2010.21484

Cardini, F., Haggard, P., \& Làdavas, E. (2013). Seeing and feeling for self and other: Proprioceptive spatial location multisensory enhancement of touch. Cognition, 127, 84-92. doi:10.1016/j.cognition.2012.12.010

Cheng, Y., Yang, C. Y., Lin, C. P., Lee, P. L., \& Decety, J. (2008). The perception of pain in others suppresses somatosensory oscillations: A magnetoencephalography study. NeuroImage, 40, 1833-1840.

Davis, M. H. (1983). Measuring individual differences in empathy: Evidence for a multidimensional approach. Journal of Personality and Social Psychology, 44, 113-126. doi:10. 1037/0022-3514.44.1.113

De Corte, K., Buysse, A., Verhofstadt, L. L., Roeyers, H., Ponnet, K., \& Davis, M. (2007). Measuring empathic tendencies: Reliability and validity of the Dutch version of the interpersonal reactivity index. Psychologica Belgica, 47, 235-260.

De Paepe, A. L., Crombez, G., Spence, C., \& Legrain, V. (2014). Mapping nociceptive stimuli in a peripersonal frame of reference: Evidence from a temporal order judgment task. Neuropsychologia, 56, 219-228.

Derbyshire, S. W., Osborn, J., \& Brown, S. (2013). Feeling the pain of others is associated with self-other confusion and prior pain experience. Frontiers in Human Neuroscience, 7(470), 1-8. doi:10.3389/ fnhum.2013.00470

Ebisch, S. J., Perrucci, M. G., Ferretti, A., Del Gratta, C., Romani, G. L., \& Gallese, V. (2008). The sense of touch: Embodied simulation in a visuotactile mirroring mechanism for observed animate or inanimate touch. Journal of Cognitive Neuroscience, 20, 1611-1623. doi:10. $1162 /$ jocn.2008.20111

Farrer, C., \& Frith, C. D. (2002). Experiencing oneself vs. another person as being the cause of an action: The neural correlates of the experience of agency. NeuroImage, 15, 596-603.
Fitzgibbon, B. M., Enticott, P. G., Rich, A. N., Giummarra, M. J., Georgiou-Karistianis, N., Tsao, J. W., ... Bradshaw, J. L. (2010a). High incidence of "synaesthesia for pain" in amputees. Neuropsychologia, 48, 3675-3678. doi:10.1016/j.neuropsychologia. 2010.07.029

Fitzgibbon, B. M., Enticott, P. G., Rich, A. N., Giummarra, M. J., Georgiou-Karistianis, N., \& Bradshaw, J. L. (2012). Mirrorsensory synaesthesia: Exploring "shared" sensory experiences as synaesthesia. Neuroscience \& Biobehavioral Reviews, 36, 645657. doi:10.1016/j.neubiorev.2011.09.006

Fitzgibbon, B. M., Giummarra, M. J., Georgiou-Karistianis, N., Enticott, P. G., \& Bradshaw, J. L. (2010b). Shared pain: From empathy to synaesthesia. Neuroscience \& Biobehavioral Reviews, 34, 500-512. doi:10.1016/j.neubiorev.2009.10.007

Gardner, W., Mulvey, E., \& Shaw, E. (1995). Regression analyses of counts and rates: Poisson, overdispersed Poisson, and negative binomial models. Psychological Bulletin, 118, 392-404.

Gillmeister, H. (2014). A new perceptual paradigm to investigate the visual remapping of others' tactile sensations onto one's own body shows "mirror touch" for the hands. Frontiers in Psychology, 5, 95. doi:10.3389/fpsyg.2014.00095

Jackson, P. L., Meltzoff, A. N., \& Decety, J. (2006). Neural circuits involved in imitation and perspective-taking. NeuroImage, 31, 429-439. doi:10.1016/j.neuroimage.2005.11.026

Karazsia, B. T., \& van Dulmen, M. H. M. (2010). Modeling infrequent outcomes: Illustrations using prospective predictors of pediatric injuries. In H. Schuster \& W. Metzger (Eds.), Biometrics: Methods, applications and analyses (pp. 1-27). New York, NY: Nova Science.

Kennett, S., Taylor-Clarke, M., \& Haggard, P. (2001). Noninformative vision improves the spatial resolution of touch in humans. Current Biology, 11, 118-1191.

Keysers, C., Kaas, J. H., \& Gazzola, V. (2010). Somatosensation in social perception. Nature Reviews Neuroscience, 11, 417428. doi: $10.1038 / \mathrm{nrn} 2833$

Keysers, C., Wicker, B., Gazzola, V., Anton, J. L., Fogassi, L., \& Gallese, V. (2004). A touching sight: SII/PV activation during the observation and experience of touch. Neuron, 42, 335-346.

Loeys, T., Moerkerke, B., De Smet, O., \& Buysse, A. (2012). The analysis of zero-inflated count data: Beyond zero-inflated Poisson regression. British Journal of Mathematical and Statistical Psychology, 65, 163-180.

Loggia, M. L., Mogil, J. S., \& Bushnell, M. C. (2008). Empathy hurts: Compassion for another increases both sensory and affective components of pain perception. Pain, 136, 168-176. doi:10.1016/j.pain. 2007.07.017

Martínez-Jauand, M., Gonzalez-Roldan, A. M., Munoz, M. A., Sitges, C., Cifre, I., \& Montoya, P. (2012). Somatosensory activity modulation during observation of others pain and touch. Brain Research, 1467, $48-55$.

McCracken, L. M. (1997). "Attention” to pain in persons with chronic pain: A behavioral approach. Behavior Therapy, 28, 271-284.

McWilliams, L. A., \& Asmundson, G. J. G. (2001). Assessing individual differences in attention to pain: Psychometric properties of the pain vigilance and awareness questionnaire modified for a non-clinical pain sample. Personality and Individual Differences, 31, 239-246.

Mirams, L., Poliakoff, E., Brown, R. J., \& Lloyd, D. M. (2010). Vision of the body increases interference on the somatic signal detection task. Experimental Brain Research, 202, 787-794. doi:10.1007/s00221010-2185-7

Morrison, I., Tipper, S. P., Fenton-Adams, W. L., \& Bach, P. (2013). "Feeling" others' painful actions: The sensorimotor integration of pain and action information. Human Brain Mapping, 34, 19821998. doi:10.1002/hbm. 22040

Mouraux, A., Diukova, A., Lee, M. C., Wise, R. G., \& Iannetti, G. D. (2011). A multisensory investigation of the functional significance 
of the "pain matrix.". NeuroImage, 54, 2237-2249. doi:10.1016/j. neuroimage.2010.09.084

Osborn, J., \& Derbyshire, S. W. G. (2010). Pain sensation evoked by observing injury in others. Pain, 148, 268-274.

Roelofs, J., Peters, M. L., McCracken, L., \& Vlaeyen, J. W. S. (2003). The pain vigilance and awareness questionnaire (PVAQ): Further psychometric evaluation in fibromyalgia and other chronic pain syndromes. Pain, 101, 299-306.

Roelofs, J., Peters, M. L., Muris, P., \& Vlaeyen, J. W. S. (2002). Dutch version of the pain vigilance and awareness questionnaire: Validity and reliability in a pain-free population. Behaviour Research and Therapy, 40, 1081-1090. doi:10.1016/S0005-7967(02)00008-6

Saxe, R., Jamal, N., \& Powell, L. (2006). My body or yours? The effect of visual perspective on cortical body representations. Cerebral Cortex, 16, 178-182.

Schaefer, M., Heinze, H. J., \& Rotte, M. (2005). Seeing the hand being touched modulates the primary somaosensory cortex. NeuroReport, $15,1101-1105$

Schaefer, M., Heinze, H. J., \& Rotte, M. (2012). Embodied empathy for tactile events: Interindividual differences and vicarious somatosensory responses during touch observation. NeuroImage, 60, 952-957. doi:10.1016/j.neuroimage.2012.01.112

Serino, A., Giovagnoli, G., \& Làdavas, E. (2009). I feel what you feel if you are similar to me. PLoS ONE, 4, e4930. doi:10.1371/journal. pone. 0004930

Serino, A., Pizzoferrato, F., \& Làdavas, E. (2008). Viewing a face (especially one's own face) being touched enhances tactile perception on the face. Psychological Science, 19, 434-438.
Spence, C., \& Driver, J. (2004). Crossmodal space and crossmodal attention. Oxford, UK: Oxford University Press.

Valentini, E., Liang, M., Aglioti, S. M., \& Iannetti, G. D. (2012). Seeing touch and pain in a stranger modulates the cortical responses elicited by somatosensory but not auditory stimulation. Human Brain Mapping, 33, 2873-2884.

Vandenbroucke, S., Crombez, G., Harrar, V., Devulder, J., Spence, C., \& Goubert, L. (2014a). Fibromyalgia patients and controls are equally accurate in detecting tactile stimuli while observing another in pain: An experimental study. Attention, Perception, \& Psychophysics, 76, 2548-2559. doi:10.3758/s13414-014-0729-9

Vandenbroucke, S., Crombez, G., Loeys, T., \& Goubert, L. (2014b). Observing another in pain facilitates vicarious experiences and modulates somatosensory experiences. Frontiers in Human Neuroscience, 8, 631. doi:10.3389/fnhum.2014.00631

Vandenbroucke, S., Crombez, G., Van Ryckeghem, D. M. L., Brass, M., Van Damme, S., \& Goubert, L. (2013). Vicarious pain while observing another in pain: An experimental approach. Frontiers in Human Neuroscience, 7, 265. doi:10.3389/fnhum.2013.00265

Vives, J., Losilla, J. M., \& Rodrigo, M. F. (2006). Count data in psychological applied research. Psychological Reports, 98, 821-835.

Völlm, B. A., Taylor, A. N. W., Richardson, P., Corcoran, R., Stirling, J., McKie, S., \& Elliott, R. (2006). Neural correlates of theory of mind and empathy: A functional magnetic resonance imaging study in a nonverbal task. Neurolmage, 29, 90-98. doi:10.1016/j.neuroimage. 2005.07.022 